\title{
Neuropsychobiology 1978;4:373-374
}

\section{Subject Index}

Affective dissorders 248, 344 Aggressivity 366 Allopurinol 188 Alpha-blocking response 150 Amino acid transport mechanisms 65 Amitriptyline 305 Analysis of EEG 207 Anticholinergic-neuroleptic antagonism 288 Antidepressant 113 Anxiety 15,283 Augmenting/reducing 150,314 Autocorrelation 93

Barbiturate sleeping time 86

Basic clinical picture 333

Benzodiazepine 283

Biochemistry 229

Biological rhythms 93

Bipolar affective disorders 276, 314

Blood

Blood groups 27

Capgras symptoms 353 Cerebral dysfunction 353 Cerebrospinal fluid 26,366

- proteins 99 Chromosomes 113 Chronicity 229 Chronic schizophrenia 360 Cinq-HİAA 150, 366 CNV 321 Compliance 121 Cortical abnormality 74

maturity 74

reactivity 74 Cycloid psychoses 276

DBH 150, 248 Deanol 140

Depression 15, 113, 180 Depressive disorders 207 Diagnostic types 288 Diazepam 36 L-Dopa 65 Dopamine 1 
Dopaminergic-cholinergic balance in

striatum 140 Dosage 283 Drug interaction 305

EEG 150, 207

in depression 207

Endogeneous depression 1,180,18

Enzymatic induction 305

Epidemiology 180

ET-495 1

Ethanol 86

Evoked potentials 321,353

Extrapyramidal syndromes 36

Familial transmission 257

i-5-HTP 65

Fluphenazine decanoate 360

enanthate 360

47, XYY, 47, XXY behavior 321

Genetic analysis of pedigrees 257

- models 257

Glucose 99
Goiter 270

Guanethidine 328

Heroin addiction 36 HLAantigens 276,344 Hormone-inhibiting factor 26

- -releasing factor 26

HumanEEG 129

HVA 150, 366

Hypothalamus 26

Hypothyroidism 270

Imipramine 113 Information processing 93

Learning and memory 129 Lithium 99, 140, 270

$\begin{array}{ll}\text { - } & \text { excretion } 328 \\ \text { - ratio } 121\end{array}$

Long-acting intramuscular neuroleptics 360 Longitudinal 180 Lundby study 180 Subject Index 
Manic-depressive illness 270

— - psychosis 65

- -melancholic psychosis 99 MAO 150

Masked 180

Median eminence 26

MIA 150

Mice strains 86

Minimal brain dysfunction 74

Monogenic models 193

Motivation 229

Motor nerve conduction 321

Neuroleptic 36

- plasma level 305

- -resistant component 288

- responsiveness 288 Neuropsychological tests 353 Neurosecretor 26 Neurotransmitters 26

Outpatient 360 Oxprenolol 328

Parietal lobes 353 Pedigree analysis 193 Pentobarbital 86 Petrie'stest 150 Physiopathology 229 Pituitary gland 26 Plasma lithium 121

- volume 328

Polygraphic recordings 366

Polymorphism 276

Polypeptides 26

Posterior slowing 74

Posture 328

Pre- and postsynaptic agonists

Probenecid test 366

Prognostic types 288

Prolonged socioenvironmental deprivation

86 Prospective 180 Psychosis 353 Pure depressive disease 333

Rating scales 99, 283 
Reaction time 93 Reactive depression 248 Receptor stimulation 1 Reciprocal inhibition 305 Red cell enzymes 276 Renin 328

Schizophrenia 65, 193, 229, 257, 288, 305

Schizophrenic process 288

Serotonin 188

Serum groups 276

Side effects 283

Soft neurological signs 74

Somatic 180

Spectral analysis 93

Spontaneous electrodermal activity (EAD)

15 S.S.AER 150 Suicide 180 Sulpiride 36

Sympathetic nervous system 328 Systemic-structural analysis of EEG 207

Tanycytes 26 Tardive dyskinesia 140 Therapeutic outcome 288 Thyroid 270 .

- antibody 270

Thyrosine 65

Thyroxine 99

Tranquilizer 283

ryptophan 65, 99

-Tryptophan 188

Ultradian periodicity 93 Urea 99

Vasopressin 129 Vigilance 93

Visual averaged evoked responses (V.AER) 150,314

perception 93

Water immersion 328 WPV 150

XYY 366 